\title{
A Mathematical Model For The Special Effects Of Phosphatidylserine On Endocrine Reaction To Reasonable Concentration Exercise In Healthy Male Subjects
}

\author{
A. Leema Rose ${ }^{\text {a }}$, A.Manickam ${ }^{\text {b }}$, Mamta Agrawal ${ }^{c}$

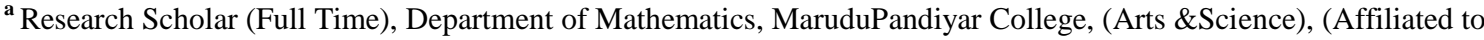 \\ Bharathidasan University, Tiruchirappalli-620 024), Vallam Post, Thanjavur-613 403, Tamilnadu, India. \\ b,c School of Advanced Sciences \& Languages, Department of Mathematics, VIT Bhopal University, Kottri Kalan (Village) - \\ 466 114), Sehore (District), Madhya Pradesh, India \\ E-mail: ${ }^{a}$ anto.leema14@gmail.com, ${ }^{\mathrm{b}}$ manickammaths2011@gmail.com, ${ }^{\mathrm{c}}$ mamta.agrwal@ vitbhopal.ac.in \\ Corresponding Author: bmanickammaths2011@gmail.com
}

Article History: Received: 10 November 2020; Revised 12 January 2021 Accepted: 27 January 2021; Published online: 5 April 2021

\begin{abstract}
In this study, we implement a three-parameter Weibull-G exponential model analysis to analyse the impact of shortrange supplementation with a modest dosage of cortisol, lactate, growth hormone and testosterone plasma attentions in healthy male profiles before, during, and after moderate intensity practice.PS supplementation facilitates a desirable athlete state of hormones through stepping up at the rates of cortisol. Ultimately, we conclude that the application part coincides with a mathematical model and the result is linked to the medical report. In the future, this paper will be very beneficial in the medicinal field.
\end{abstract}

Keywords: Cortisol, Exponential distribution, Generalized Exponential, GH, Placebo

Mathematical subject classification: [62H]_(xx );62[NO $]_{-}(5) ; 90 \mathrm{~B} 25$.

\section{Introduction}

The exponential distribution (ED) [3] has a variety of uses from approaches with paths to life science, performance assessment, basic research and clinical trials. This approach is a specific example Weibull's two variables distribution with a parameter of form equal to 1 . The derivation of this distribution and other features can be establish in [2]. It is said that a random variable $\mathrm{Y}$ has an exponential distribution (ED) with parameters $\delta>$ 0 if its probability density function (pdf) is

$$
g(y)=\delta e^{-\delta y}, y>0
$$

When the cumulative distribution function (cdf) is

$G(y)=1-e^{-\delta y}, y>0$.

The formula gives the survival function

$S(y)=1-G(y)=e^{-\delta y}, y>0$.

And the hazard function is

$h(y)=\delta$

The implementation of the Weibull distribution [6] is a common distribution with monotonous failure tariffs for modelling phenomenon. As a result, an amount of innovative distributions that better model the data constructed in the literature as Weibull distribution ramifications. We took the reported data (Banerjee et al., 2013) in the present paper and analysed it using Weibull-G exponential distribution

\section{Mathematical Model}

A parameters are three Weibull-G exponential model has been deliberate in this segment. The Weibull-G exponential distribution's, cumulative distribution function (cdf) is known by

$$
\begin{aligned}
& F(y ; p, q, \delta)=1-e^{-p\left[e^{\delta y}-1\right]^{q}}, \quad p, q, \delta>0 \\
& \text { The pdf equivalent to Eq. (2.1) is } \\
& \qquad f(y ; p, q, \delta)=p q \delta e^{\delta y}\left[e^{\delta y}-1\right]^{q-1} e^{-p\left[e^{\delta y}-1\right]^{q}}, \\
& \begin{array}{l}
y>0, \quad(2.2) \\
\text { Where } p, q>0 \text { and } \delta>0 \text { are two another shape parameters. }
\end{array}
\end{aligned}
$$


We represent using $Y \sim W G E D(p, q, \delta)$ a random variable taking the pdf Eq.(2.1). The survival function $S(y)$, hazard rate function $h(y)$, reversed hazard rate function $r(y)$ and cumulative hazard rate function $H(y)$ of $\mathrm{Y}$, are known by

$$
\begin{aligned}
& y>0, \\
& y>0, \\
& \begin{array}{r}
y \\
\quad \text { and }
\end{array}
\end{aligned}
$$

$$
\begin{gathered}
S(y ; p, q, \delta)=1-F(y ; p, q, \delta)=e^{-p\left[e^{\delta y}-1\right]^{q}}, \\
h(y ; p, q, \delta)=p q \delta e^{\delta y}\left[e^{\delta y}-1\right]^{q-1}, \\
r(y ; p, q, \delta)=\frac{p q \delta e^{\delta y}\left[e^{\delta y}-1\right]^{q-1} \cdot e^{-p\left[e^{\delta y}-1\right]^{q}}}{1-e^{-p\left[e^{\delta y}-1\right]^{q}},} \\
H(y ; p, q, \delta)=\int_{0}^{\infty} h(y ; p, q, \delta) d x=p\left[e^{e^{\delta y}}-1\right]^{q},
\end{gathered}
$$

respectively.

\section{Applications}

The perseverance of this study was to explore the short-range stimulation stimulus with a small dose of phosphatidylserine on cortisol, lactate, growth hormone and testosterone plasma attentions in 10 active male topics before, during and after medium strength exercise. Each topic was assigned to use a double-blind, placebomeasured crossover project to consume $600 \mathrm{mg}$ PS or placebo per day for 10 days. During the 21-day analysis, each participant completed three test periods. A graded exercise test was conducted by the participants.

The second and third activity cycles were completed during exercise and recovery to identify reactions in order to assert reassuring pressure during breakage. The participant was hypothetical to drink liquid ad libitum during the remainder of the implementation and retrieval segment of the training. Supplements for PS and placebo are controlled throughout the chewable strawberry-flavoured medicinal products system obtained from SwissCo Development AG.

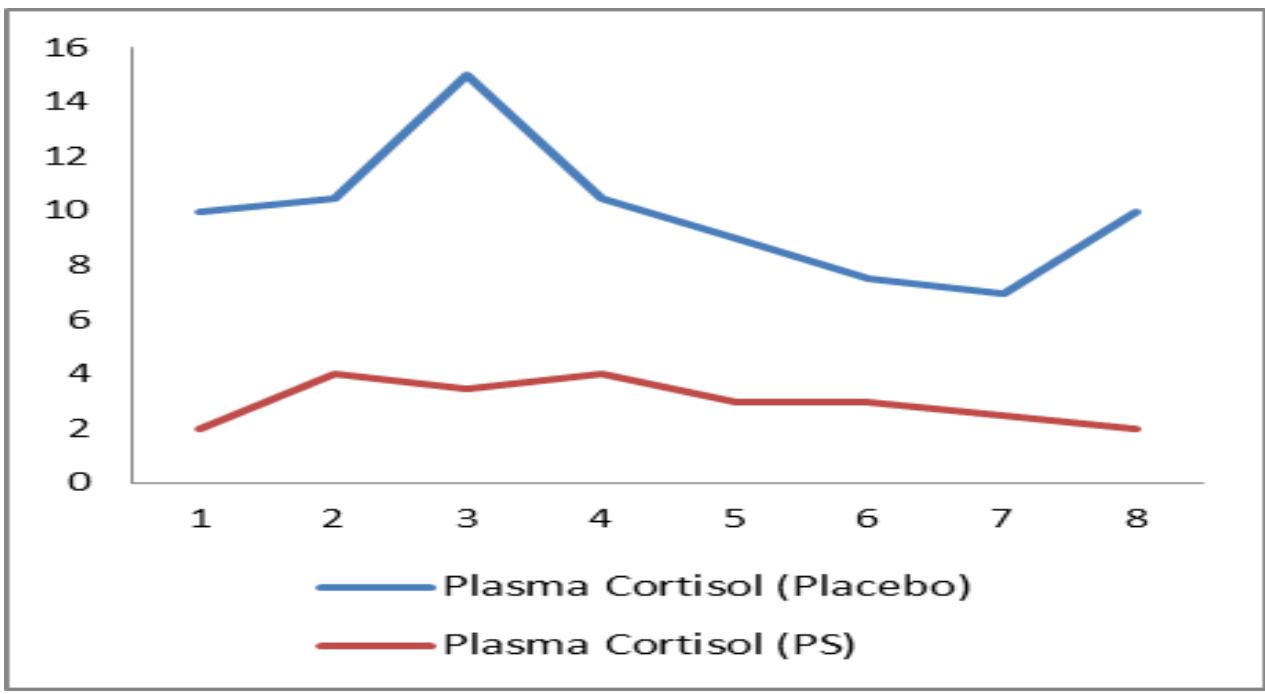



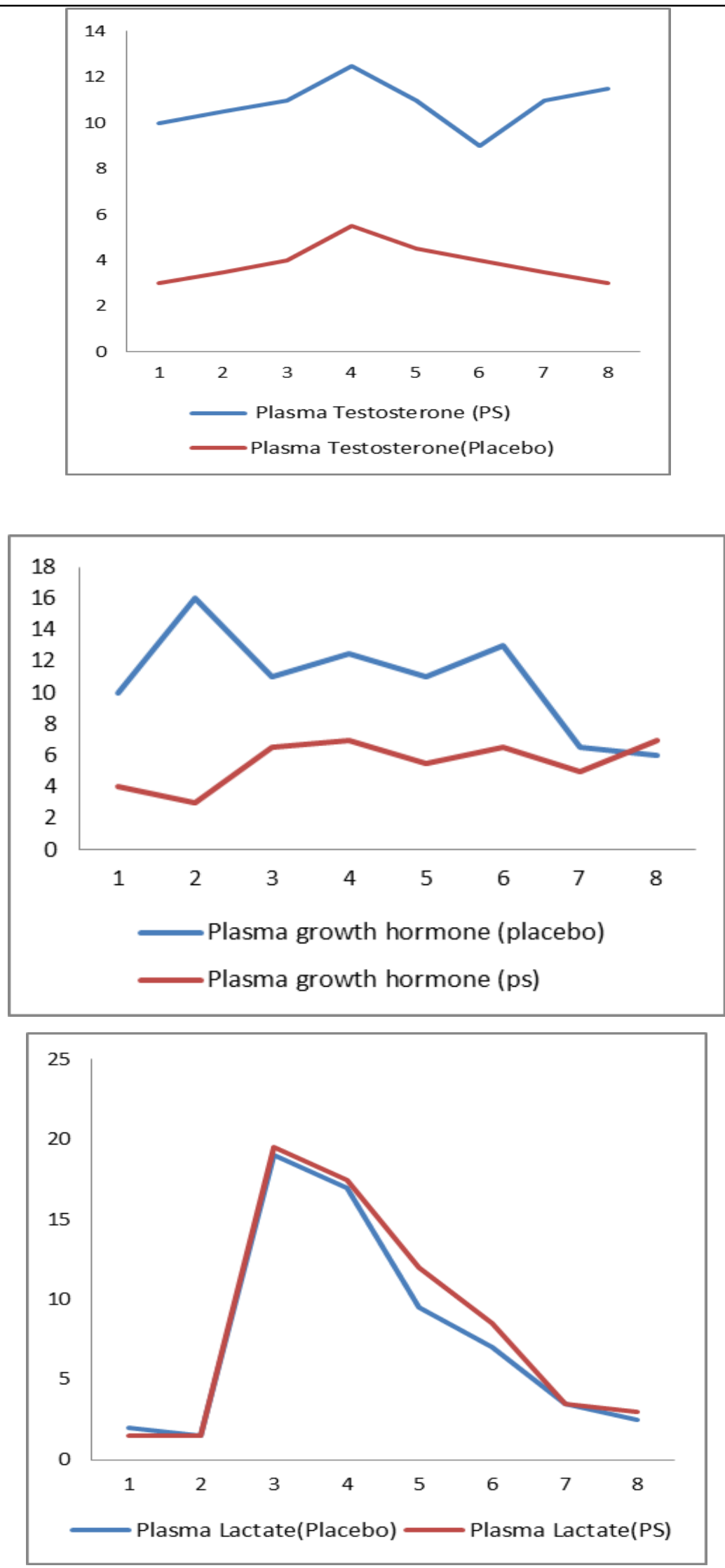

Figure 3.1.Following 10 days of oral therapy with $600 \mathrm{mg} \mathrm{S}-\mathrm{PS}$ or placebo, Cortisol, testosterone, lactate and growth hormone reaction to exercise. 
Its illustrations the result of S-PS and placebo treatment at $0,10,20,30,40,50$, and 60 minutes after cortisol, testosterone, lactate and growth hormone exercise. Concentrations of plasma testosterone increased as compared to placebo; $[2,8]$. Exercise in both groups caused an increase in lactate rates but failed to reach statistical implications. Supplementation could not take placevariances in lactate reaction among the S-PS and placebo groups. However, supplementation could not affect the plasma growth hormone rates reaction trend. Area under the arc study showed substantial variances among S-PS and placebo cortisol. Growth hormone [1, 5] replies speculated tiny and were not suggestively dissimilar between them. Workout initiated increases in plasma lactate attentions in the course of both trials and supplementation did not disturb these attentions; consequently, during both stage of the study, the participants exercised related capacities.

\section{Mathematical results}
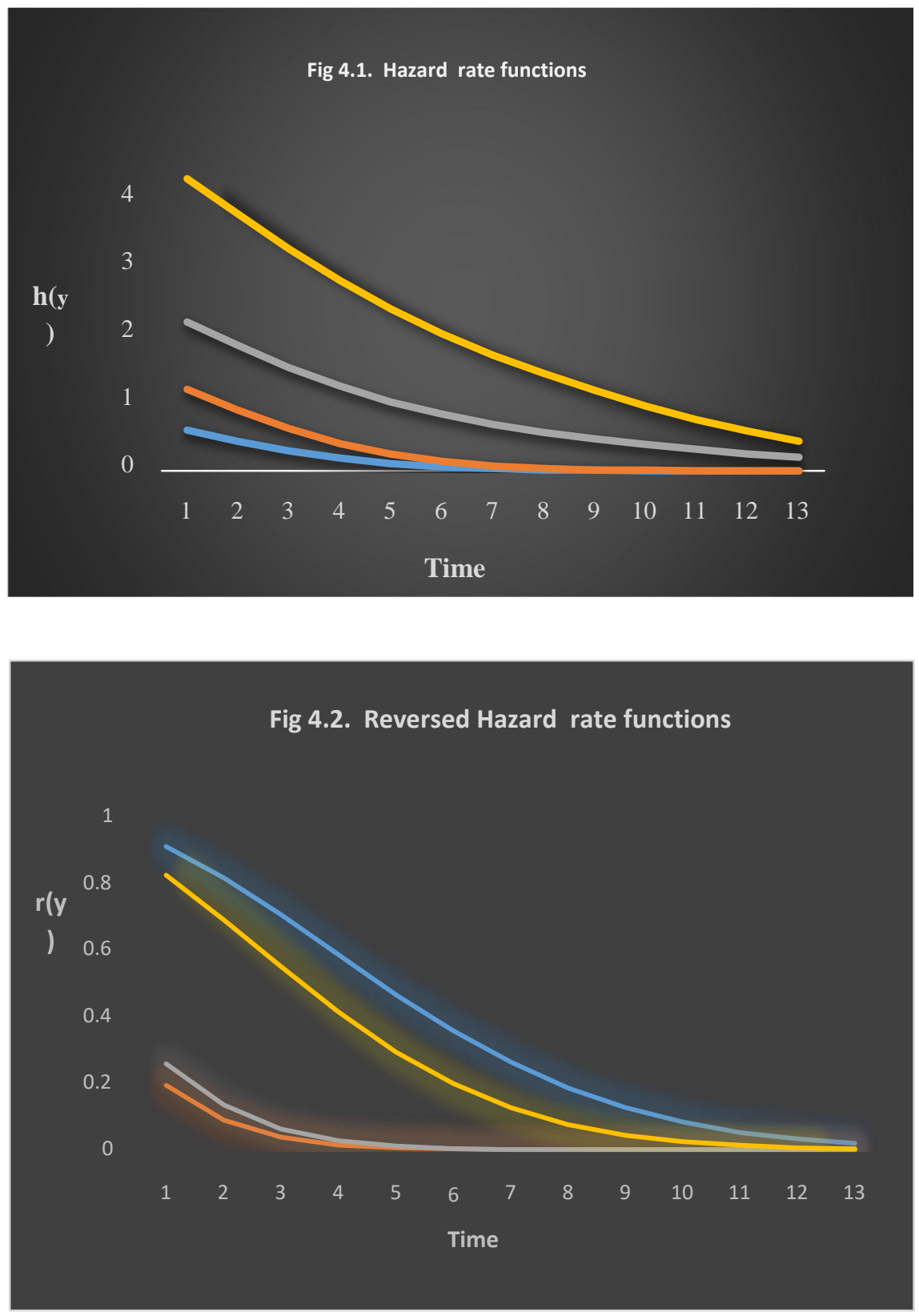

\section{Conclusions}

We used the exponential distribution model of Weibull-G in this analysis to analyse the plasma growth hormone profiles of female rats. For the selected medical data, we have plotted probability density function, probability survival function, hazard rate function and reverse hazard rate function. We can assume that the exponential distribution model of Weibull-G is well suited for mathematical analysis of medical data. It is useful to practitioners in the medical field. 


\section{Acknowledgements}

The authors would like to thank the National Institute of Medical Science, IISC Bangalore for the use of ANSYS software

\section{Conflict of Interests}

The authors declare that there is no conflict of interests.

\section{References}

Fahey TD, Pearl M: The hormonal and perceptive effects of phosphatidylserine administration during two weeks of resistive exercise -induced overtraining. Biol Sport 1998, 15:135-144.

Fry AC, Kraemer WJ, Ramsey LT: Pituitary-adrenal-gonadal responses to highintensity resistance exercise overtraining. J Appl Physiol 1998, 85(6):2352-2359.

Gupta, R. D. and Kundu, D. (2001). Exponentiated exponential family; an alternative to gamma and Weibull. Biometrical journal, Vol. 43, pp. 117-130.

Gupta, R. D. and Kundu, D. (2003). Discriminating between the Weibull and the GE distributions. Computational Statistics and Data Analysis, Vol. 43, pp. 179-196.

Monteleone P, Maj M, Beinat L, Natale M, Kemali D: Blunding by chronic phosphatidylserine administration of the stress induced activation of the hypothalamopituitary-adrenal axis in healthy men. Eur Clin Pharmacol 1992, 42:385-388.

Weibull, W. (1951). Wide applicability. Journal of applied mechanics, Vol. 40, pp. 203210.

Welsh TH Jr, Bambino TH, Hsueh AJ: Mechanism of glucocorticoid-induced suppression of testicular androgen biosynthesis in vitro. Biol Reprod 1982, 27(5): 1138-1146.

Wheeler GD, Wall SR, Belcastro AN , Cumming DC: Reduced serum testosterone and prolactin levels in male distanc runners. JAMA 1984, 252(4): 514-516.

Wittert GA, Stewart DE, Graves MP, Ellis MJ, Wells JE, Donald RA, Espiner EA: Plasma corticotrophin releasing factor and vasopressin responses to exercise in normal man. Clin Endocrinol (Oxf) 1991, 35(4):311317. 